This item was submitted to Loughborough's Research Repository by the author.

Items in Figshare are protected by copyright, with all rights reserved, unless otherwise indicated.

\title{
Tailoring the AMC and EBG characteristics of periodic metallic arrays printed on grounded dielectric substrate
}

PLEASE CITE THE PUBLISHED VERSION

http://dx.doi.org/10.1109/TAP.2005.861575

PUBLISHER

(C) Institute of Electrical and Electronics Engineers (IEEE)

VERSION

VoR (Version of Record)

LICENCE

CC BY-NC-ND 4.0

\section{REPOSITORY RECORD}

Goussetis, George, Alexandros P. Feresidis, and J.C. Vardaxoglou. 2019. "Tailoring the AMC and EBG Characteristics of Periodic Metallic Arrays Printed on Grounded Dielectric Substrate”. figshare. https://hdl.handle.net/2134/9423. 
This item was submitted to Loughborough's Institutional Repository (https://dspace.lboro.ac.uk/) by the author and is made available under the following Creative Commons Licence conditions.

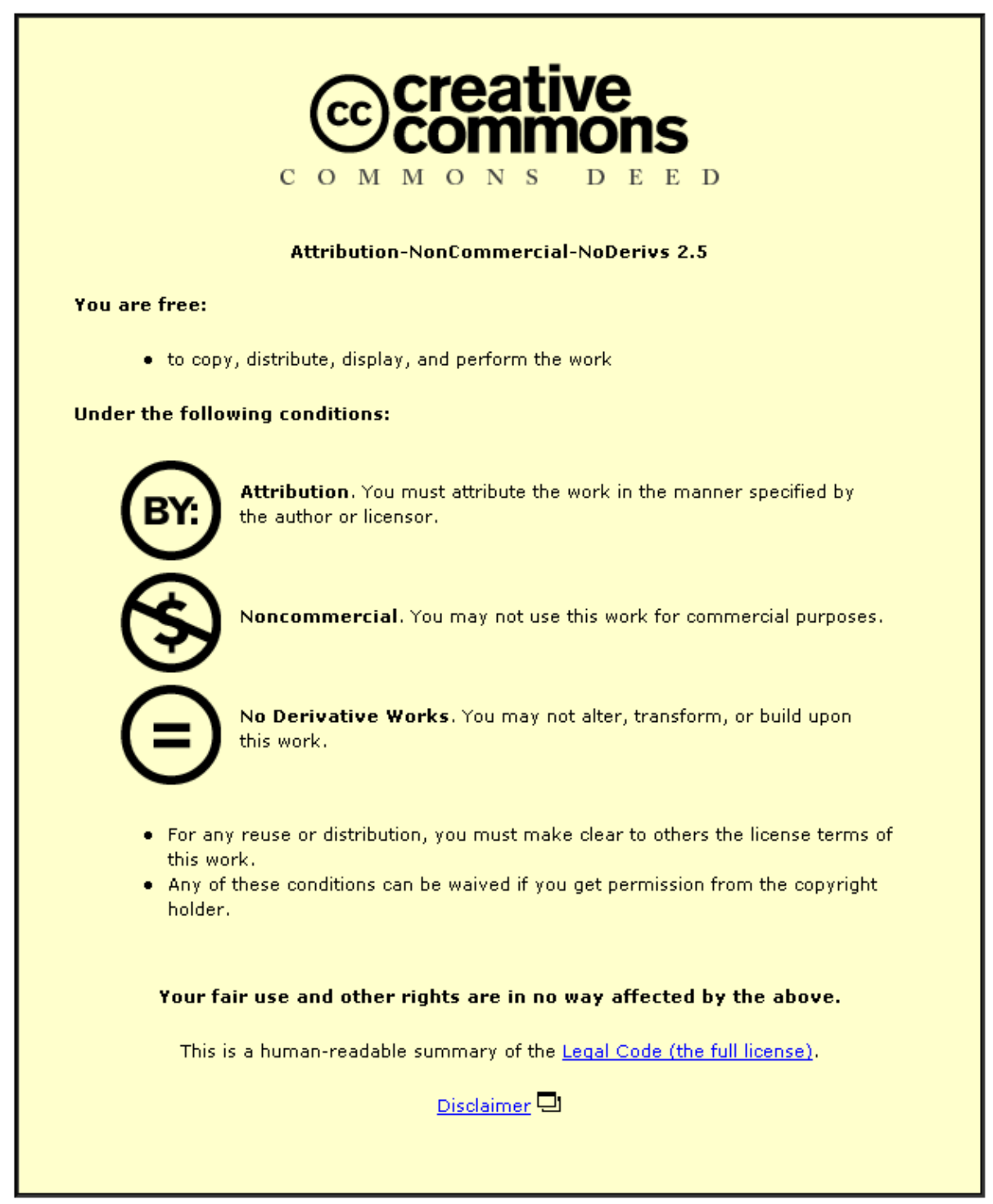

For the full text of this licence, please go to: http://creativecommons.org/licenses/by-nc-nd/2.5/ 


\title{
Tailoring the AMC and EBG Characteristics of Periodic Metallic Arrays Printed on Grounded Dielectric Substrate
}

\author{
George Goussetis, Member, IEEE, Alexandros P. Feresidis, Member, IEEE, and \\ John (Yiannis) C. Vardaxoglou, Member, IEEE
}

\begin{abstract}
The artificial magnetic conductor (AMC) and electromagnetic band gap (EBG) characteristics of planar periodic metallic arrays printed on grounded dielectric substrate are investigated. The currents induced on the arrays are presented for the first time and their study reveals two distinct resonance phenomena associated with these surfaces. A new technique is presented to tailor the spectral position of the AMC operation and the EBG. Square patch arrays with fixed element size and variable periodicities are employed as working examples to demonstrate the dependence of the spectral AMC and EBG characteristics on array parameters. It is revealed that as the array periodicity is increased, the AMC frequency is increased, while the EBG frequency is reduced. This is shown to occur due to the different nature of the resonance phenomena and the associated underlying physical mechanisms that produce the two effects. The effect of substrate thickness is also investigated. Full wave method of moments (MoM) has been employed for the derivation of the reflection characteristics, the currents and the dispersion relations. A uniplanar array with simultaneous AMC and EBG operation is demonstrated theoretically and experimentally.
\end{abstract}

Index Terms-Artificial magnetic conductor (AMC), electromagnetic band gap (EBG), frequency selective surface (FSS), metamaterials.

\section{INTRODUCTION}

$\mathbf{O}$ VER the last few years there has been increased research interest in electromagnetic band gap (EBG) structures and their applications in microwave and (sub)millimeter wave frequencies [1]. Applications of EBG structures include surface wave suppression [2]-[5], improved antennas for modern wireless communication systems [5]-[7], and novel microwave circuits and waveguides [7], [8]. Recently, metallo-dielectric high impedance surfaces (HIS) have been presented, that upon illumination from an incident wave perform as equivalent artificial magnetic conductors (AMC) [2]. Such a structure fully reflects incident waves with a zero degrees reflection phase and is complimentary to an electric conductor (which introduces a phase shift of $180^{\circ}$ ). AMC surfaces have found numerous applications as ground planes in printed antennas [2], [9], [10], low profile cavity antennas [11], [12] and as reflectors for aperture coupled patch antennas [13].

Manuscript received May 26, 2005. This work was supported in part by the U.K. EPSRC under Grant EP/C510607/1.

The authors are with the Wireless Communications Research group, Department of Electronic and Electrical Engineering, Loughborough University, Leicestershire LE11 3TU, U.K. (e-mail: G.Goussetis@lboro.ac.uk; a.feresidis@ieee.org).

Digital Object Identifier 10.1109/TAP.2005.861575

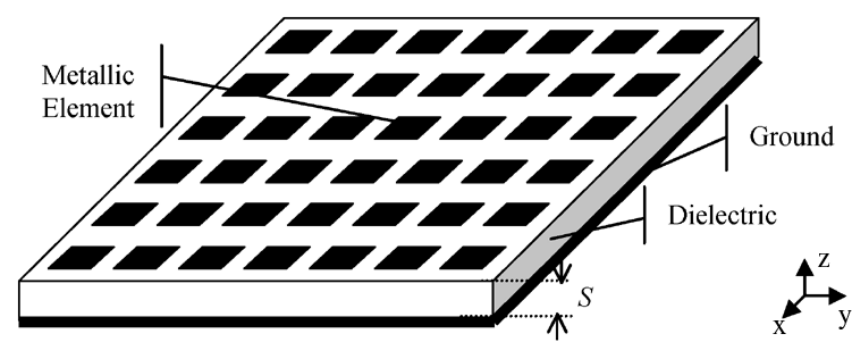

(a)

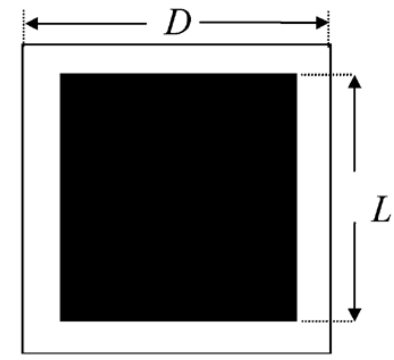

(b)

Fig. 1. Layout of the structure under investigation (a) bird's eye view (b) unit cell.

The geometry of the first HIS reported in [2] consists of a periodic metallic patch array printed on a metal-backed (grounded) dielectric slab with metallic vias connecting the patches to the ground. The presence of vias in this mushroom-type structure imposes an electromagnetic band gap at the same frequency range as the AMC property. In other words, the mushroom structure exhibits high surface impedance for both normally incident and surface waves at the same frequency band. Hence, it reflects a normally incident plane wave with zero phase shift, therefore behaving as an AMC, and at the same frequency does not support surface waves, therefore behaving as an EBG.

Grounding vias complicate the fabrication of AMC surfaces, particularly at upper microwave and millimeter-wave frequencies. In order to simplify the fabrication, several research groups have, in the last five years, worked on implementing AMC surfaces without vias in a completely planar (also mentioned as "uniplanar" throughout this paper) configuration [9]-[13]. These structures are essentially periodic frequency selective surfaces (FSS) [14], [15], printed on grounded dielectric slabs, as shown in Fig. 1. Previous work has focused on providing insight on the operation of the AMC surfaces without vias [12], [13]. In [16], a fast and accurate method for band char- 


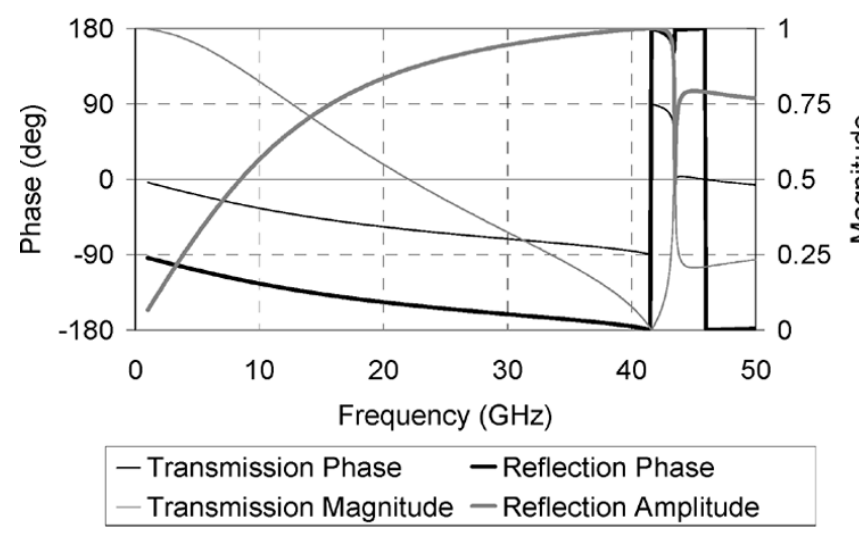

(a)

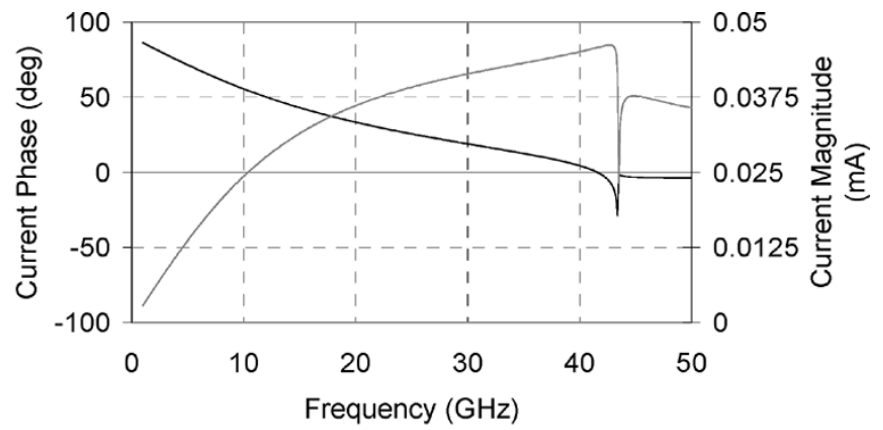

- Current Phase

(b)

Fig. 2. Plane wave normally incident on free-standing square patch FSS with $D=6.9 \mathrm{~mm}$ and $L=6.1 \mathrm{~mm}$ : (a) transmission and reflection response and (b) currents excited on the elements.

acterization of such surfaces through a network synthesis has been proposed and it has been demonstrated that in the absence of vias, the EBG does not normally coincide with the AMC frequency band. This can deteriorate the benefits of AMC surfaces in certain applications, where surface wave suppression is advantageous.

In this paper we study the AMC and EBG characteristics of uniplanar periodic metallic arrays printed on grounded dielectric substrate and we present a method to control the spectral position of these two properties. In particular, we investigate the effect of the array parameters on both the EBG and AMC frequency bands and we show that we can tailor the spectral position of the two properties almost independently by altering the periodicity of a square patch array. From a practical point of view, this allows the flexibility to design uniplanar arrays with either one or both of these properties, depending on the requirements of the application.

The currents induced on the elements of the metallic array in close proximity to a ground plane under plane wave illumination are studied for the first time. Based on this study, two distinct resonant phenomena are observed which lead to conclusions regarding the nature of the AMC and EBG properties. It is demonstrated that the AMC operation is not directly related to the resonance of the FSS array. In fact, it is revealed that with varying array periodicity, the AMC band moves opposite to the FSS resonance in frequency. On the contrary, it is shown that the EBG frequency follows the trend of the FSS resonance. A further study on the effect of substrate thickness is also presented. Based on the conclusions drawn from the above studies, a uniplanar surface with both EBG and AMC operations in the same frequency band is designed and tested experimentally.

\section{Resonance Phenomena of FSS in Proximity to A GROUND Plane}

\section{A. Free-Standing FSS}

FSSs have been studied for many years and their behavior is well understood [15]. For an array consisting of conducting elements under plane wave incidence, a maximum current magnitude is excited on the elements at the array resonant frequency. The current in this case is in phase with the incident field, i.e., the

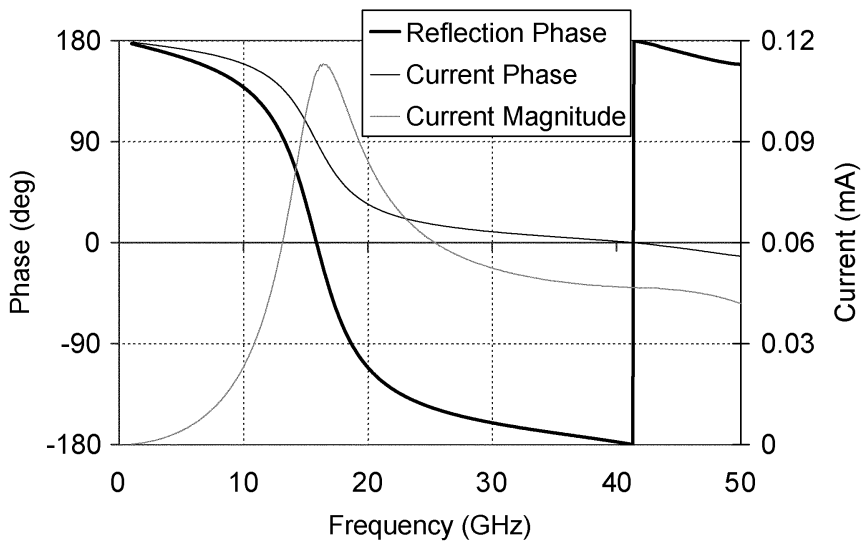

Fig. 3. Plane wave normally incident on square patch FSS with $D=6.9 \mathrm{~mm}$ and $L=6.1 \mathrm{~mm}$ at distance $S=1.13 \mathrm{~mm}$ from a ground plane: reflection phase for incident plane wave and currents excited on the elements.

impedance seen by the incident wave is purely ohmic (real), since the capacitive and inductive parts cancel out. As a result of the resonance, the incident wave is reflected with a phase reversal.

The above are briefly demonstrated here by means of an example based on a free-standing square patch FSS with dimensions $D=9.9 \mathrm{~mm}$ and $L=6.1 \mathrm{~mm}$. Full wave simulation results are obtained using a Floquet modal analysis of the unit cell, which leads to the formulation of an integral equation (IE) and its solution using Galerkin method of moments (MoM) [15]. The method has been extensively described in the literature and is known to be a fast and accurate technique for the characterization of such structures. Fig. 2(a) shows the transmission and reflection response of the capacitive FSS illuminated with a normally incident plane wave. Fig. 2(b) shows the magnitude and phase of the currents excited on the elements, assuming incident field of magnitude $1 \mathrm{~V} / \mathrm{m}$. The array resonance occurs at $41.5 \mathrm{GHz}$, where the current phase is $0^{\circ}$.

\section{B. FSS in Proximity to a Ground Plane}

For FSS surfaces in close proximity to a ground plane some subtle differences emerge. Due to the ground plane, incident waves are fully reflected at all frequencies. However in this type of structure, careful investigation reveals that two distinct resonant phenomena occur for a normally incident wave. 


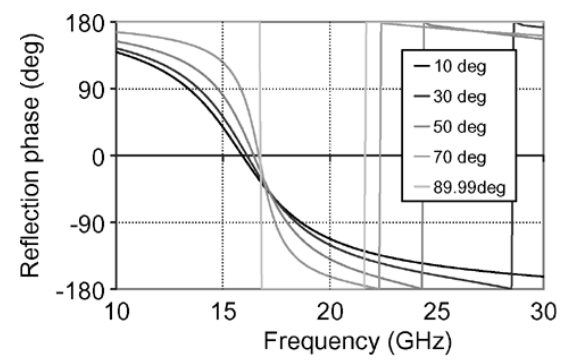

(a)

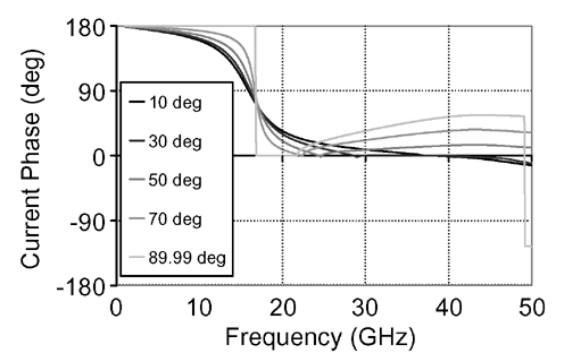

(b)

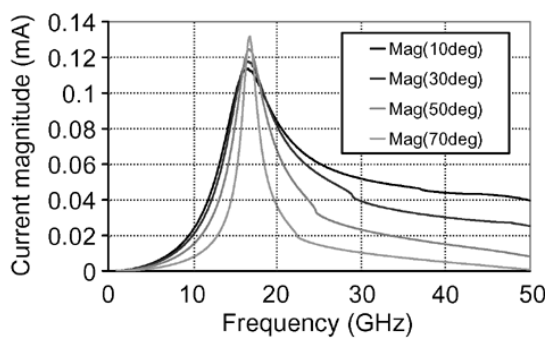

(c)

Fig. 4. (a) Reflection phase response, (b) current phase, and (c) magnitude for the array of Fig. 3 and for varying incident angles.

As before, we can identify the array resonance at the frequency where the currents excited on the array are in phase with the incident wave (i.e., zero current phase). At this frequency, the incident wave is reflected from the FSS array with a phase reverse, as in the case of the free-standing FSS resonance. However it can be found that there also occurs a Fabry-Perot type of resonance at the cavity formed between the ground plane and the FSS [12], [17], [18]. The Fabry-Perot resonance occurs at frequencies different than the array resonance. This strong cavity-type resonance excites maximum currents on the elements (which in general are out of phase with the incident wave) and the incident wave is reflected with a zero phase shift.

These resonance phenomena are demonstrated by means of an example. The FSS array of Fig. 2 is considered at distance $S=1.13 \mathrm{~mm}$ from an infinite ground plane. The structure is initially illuminated with a normally incident plane wave of amplitude $1 \mathrm{~V} / \mathrm{m}$. Fig. 3 shows the reflection phase of the normally incident plane wave and the excited current (magnitude and phase) on the elements. As in the free-standing case, the array resonance is identified by the zero current phase. This resonance shares the same characteristics with the resonance of the free-standing case. It occurs at the same frequency and the currents excited are of equal magnitude and phase to the current excited in the free-standing case [Fig. 2(b)]. However at around $15.9 \mathrm{GHz}$ the Fabry-Perot resonance occurs, indicated by the nearly maximum current magnitude excited on the elements. The current phase however is not zero but around $90^{\circ}$, i.e., a capacitive phase of the FSS array is observed indicating that the FSS array itself is not at resonance. The normally incident wave sees a high surface impedance (open circuit) and is reflected with zero reflection phase.

As the angle of the TE incident plane wave moves from normal to grazing incidence, the frequency characteristics of the two resonances (Fabry-Perot and array resonance) vary. Fig. 4(a) shows the reflection phase response of the structure of Fig. 3 as the incident angle varies from $10^{\circ}$ (close to normal incidence) to $89.99^{\circ}$ (almost surface wave). The current phase and magnitude are shown in Fig. 4(b) and (c), respectively. Note that while the Fabry-Perot resonant frequency remains nearly constant, the array resonance shifts to lower frequencies.

At $90^{\circ}$ incidence the plane wave response is not a valid description any more. Instead the structure is characterized by the dispersion relation of surface waves along the contour of the Brillouin zone, shown in Fig. 5. The MoM formulation for the derivation of the dispersion diagrams is similar as for normal

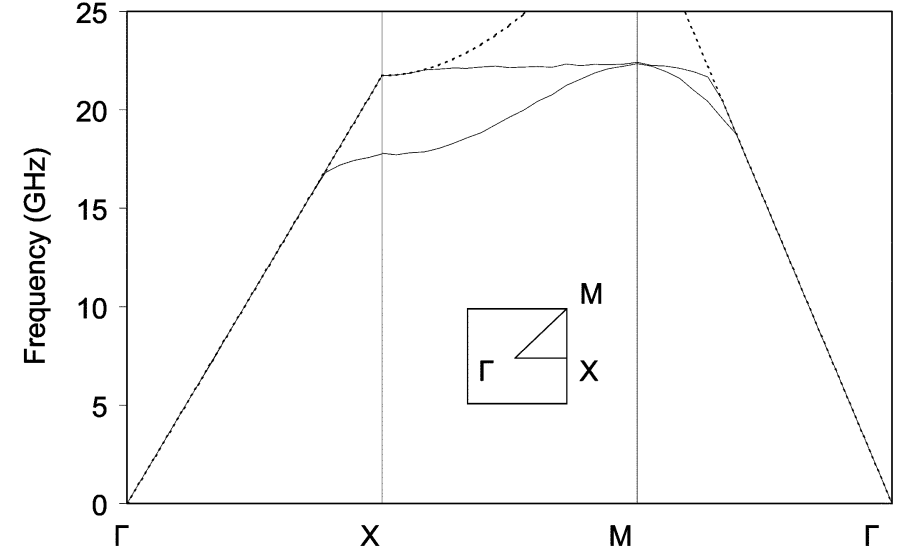

Fig. 5. Dispersion relation for the array of Fig. 3.

incidence, only now the excitation is set to zero, the tangential wavenumbers are set to correspond to a particular point on the $x$-axis of the dispersion diagram and the eigenfrequencies are specified so that the homogenous problem accepts real solutions [19]. A TE band gap emerges along the $\Gamma \mathrm{X}$ direction at about $17.5 \mathrm{GHz}$. This frequency corresponds with a very good accuracy to the frequency where the array resonates for $89.99^{\circ}$ incidence [zero current phase in Fig. 4(b)]. This is a good indication that the array resonance is the underlying physical mechanism of the EBG.

\section{C. $A M C$ and EBG Operation}

Based on the above study, we can now relate each of the AMC and EBG properties with one of the two distinct resonant phenomena observed. The AMC operation emerges by virtue of the resonance of the cavity formed between the periodic array and the ground plane. To a ray optics approximation, the cavity resonance critically depends on the thickness, $S$, of the cavity and the value of the transmission phase $\phi_{T}$ provided by the FSS array, according to the following resonance condition:

$$
\phi_{T}=k_{z} \cdot S-(2 N+1) \cdot \frac{\pi}{2}, \quad N=0,1,2 \ldots
$$

where $k_{z}$ is the propagation constant along the normal to the surfaces. Equation (1) is derived from ray optics, and hence is more accurate for the higher values of $N(N \geq 1)$ but the basic physics are the same for a thin AMC $(N=0)$.

The EBG emerges as a result of the array resonance and the array periodicity. The surface waves in a periodic structure 


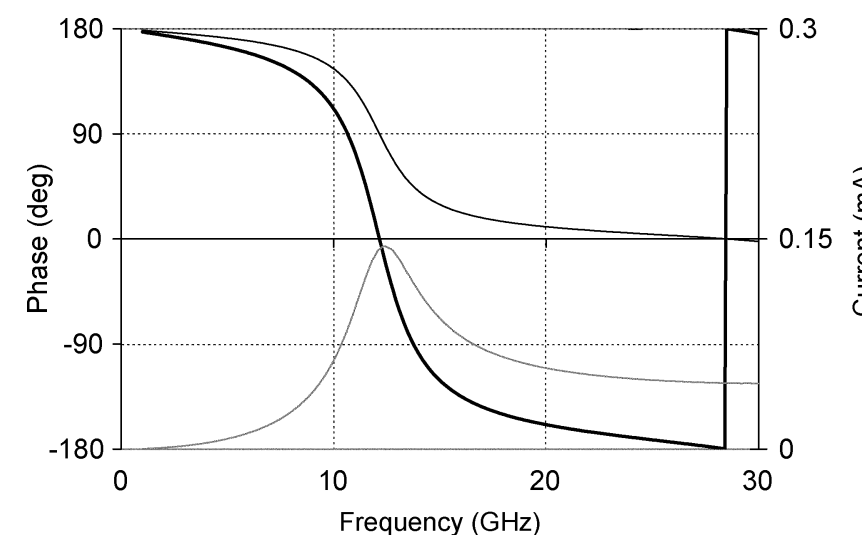

(a)

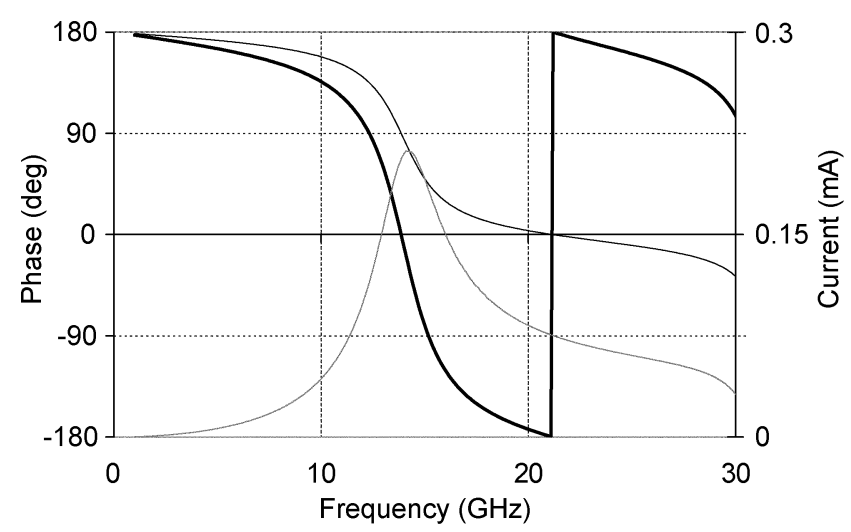

(c)

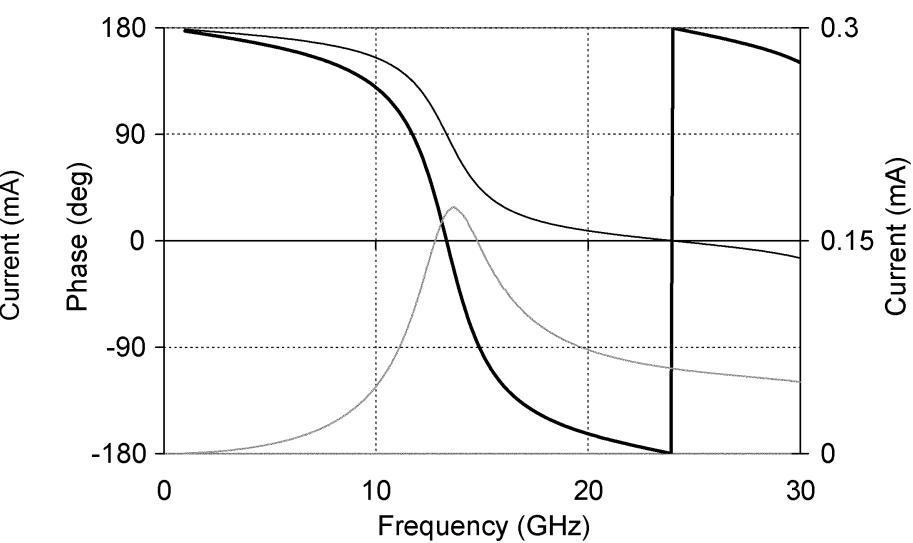

(b)

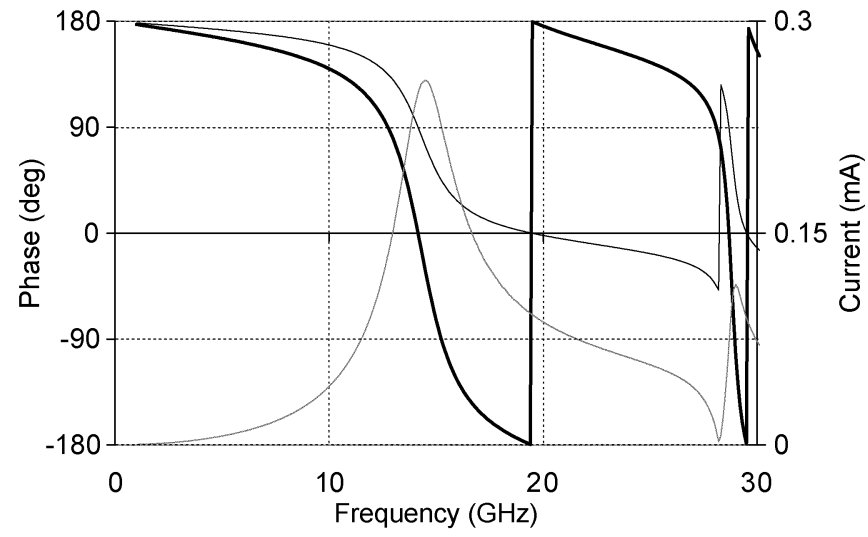

(d)

Fig. 6. Plane wave normally incident on square patch FSS with (a) $D=6.9 \mathrm{~mm}$, (b) $D=7.9 \mathrm{~mm}$, (c) $D=8.9 \mathrm{~mm}$, (d) $D=9.9 \mathrm{~mm}$ and $L=6.1 \mathrm{~mm}$ printed on grounded dielectric of thickness $\mathrm{S}=1.13 \mathrm{~mm}$ and $\varepsilon_{\mathrm{r}}=2.2$ : reflection phase for normally incident plane wave (thick black line) and current excited on the elements; thin black line: phase, thin grey line: magnitude.

below the bandgap are a superposition of travelling and standing waves [20]. At the lower EBG cutoff frequency, the half guided wavelength becomes equal to the periodicity, $D$, and a standing wave is formed. Successive elements are in this case out of phase and a bandgap emerges. At the higher cutoff of the bandgap, there is a similar standing wave, only now the spatial locations of energy maximums and nulls are interchanged. The upper cutoff frequency is typically defined by the periodicity of the lattice. The EBG bandwidth occurs due to the variation of the spatial location of energy concentration in these two limit cases.

\section{TAILORING THE EBG AND AMC CHARACTERISTICS USING THE ARRAY PERIODICITY}

In this section we study the AMC and EBG properties of a practical structure consisting of a capacitive FSS over a grounded dielectric substrate (Fig. 1). An absolute TE/TM bandgap occurs. It is demonstrated that in the absence of vias, the AMC and EBG bands do not in general coincide in the frequency domain. A method to tailor the spectral position of the two properties using the array periodicity is presented. We have considered an array of square patches of size $L=6.1 \mathrm{~mm}$, and square unit cell (Fig. 1). The dielectric substrate parameters are $S=1.13 \mathrm{~mm}, \varepsilon_{r}=2.2$. Four different arrays are studied with periodicities $(D)$ 6.9, 7.9, 8.9 , and $9.9 \mathrm{~mm}$ respectively. In all four cases, the grating lobe region lies well above the resonance of the array.

\section{A. AMC Characteristics}

Fig. 6 shows the reflection phase of a normally incident plane wave of strength $1 \mathrm{~V} / \mathrm{m}$ for the four structures under consideration. The magnitude and phase of the currents excited on the elements are also shown. The smallest periodicity, $6.9 \mathrm{~mm}$, yields an AMC frequency of $12.1 \mathrm{GHz}$, which increases to $14.1 \mathrm{GHz}$ for the largest periodicity, $9.9 \mathrm{~mm}$. This trend is in contrast to the array resonance, which is indicated by the zero current phase in Fig. 4 and reduces as the periodicity of the array increases. Therefore the spectral separation between the AMC frequency and the array resonance reduces as the periodicity of the metallic array increases (Fig. 6). This observation is important for our discussion later on.

As in Fig. 3, the current levels at the Fabry-Perot resonance are nearly three times higher than those in the array resonance. The current magnitude levels are lower for more closely packed elements. According to Lenz's law in a quasistatic approach, this is due to the increasing opposing mutual inductance between neighbor co-linear elements with co-phase currents.

\section{B. Electromagnetic Band Gap Characteristics}

Fig. 7 shows the dispersion curves for the four structures under consideration. Only bounded surface wave modes are presented. Due to the presence of the dielectric, TM modes have a stronger horizontal electric field component and a TM 


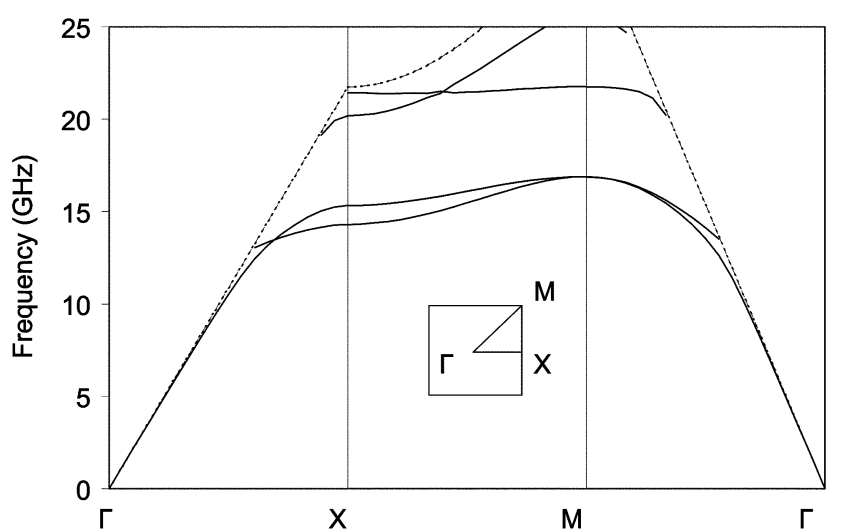

(a)

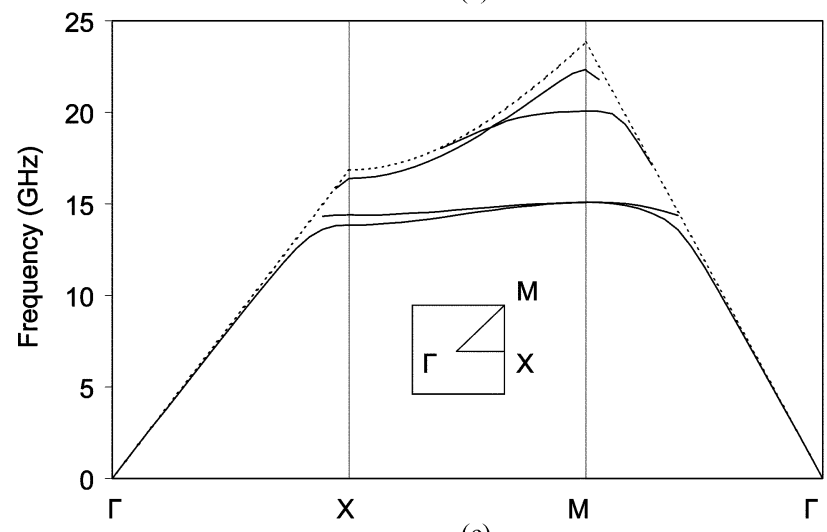

(c)

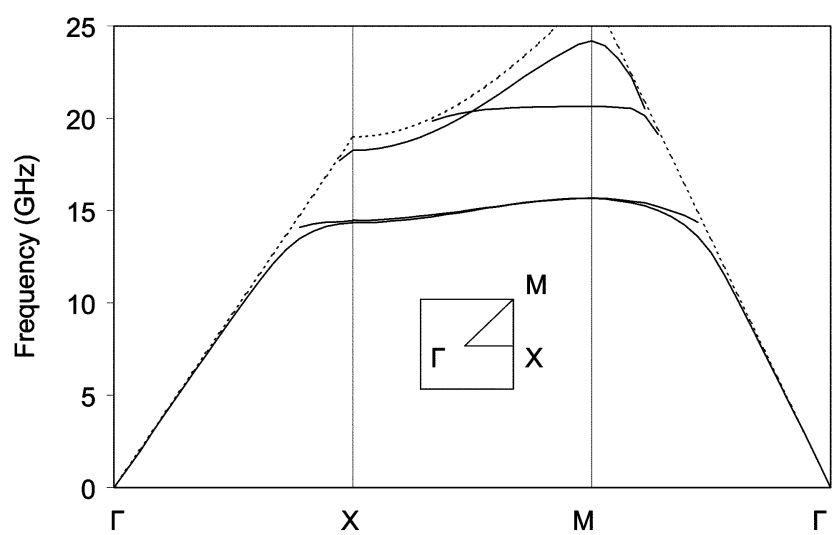

(b)

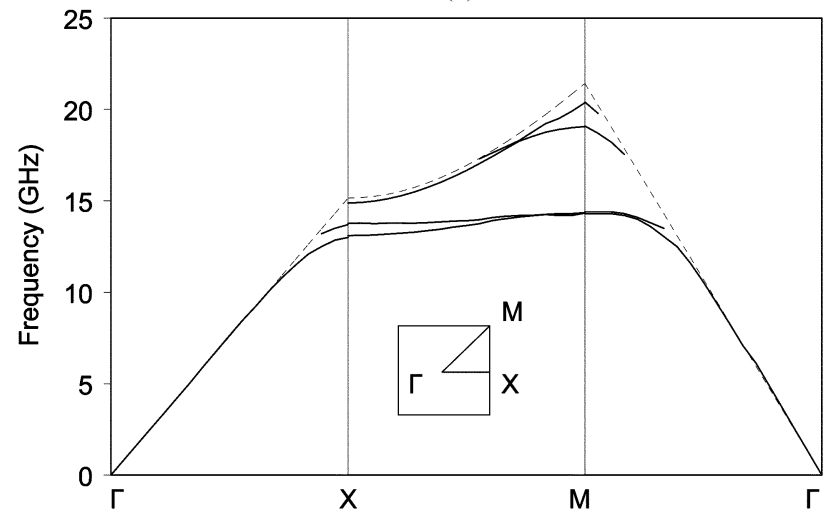

(d)

Fig. 7. Dispersion relation for square patch FSS with (a) $D=6.9 \mathrm{~mm}$, (b) $D=7.9 \mathrm{~mm}$, (c) $D=8.9 \mathrm{~mm}$, (d) $D=9.9 \mathrm{~mm}$ and $L=6.1 \mathrm{~mm}$ printed on grounded dielectric of thickness $\mathrm{S}=1.13 \mathrm{~mm}$ and $\varepsilon_{\mathrm{r}}=2.2$.

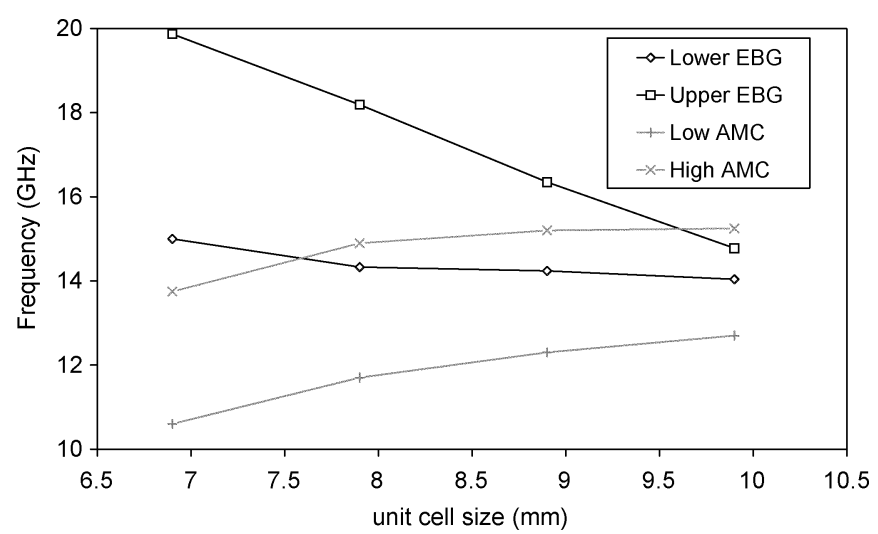

Fig. 8. Lower and upper frequency for EBG and AMC performance for square patch array $(\mathrm{L}=6.1 \mathrm{~mm})$ with varying unit cell.

bandgap also emerges in the $\Gamma X$ direction. As shown, the common TE/TM surface wave bandgap cutoff drops from around $15 \mathrm{GHz}$ for array periodicity $6.9 \mathrm{~mm}$ to around 13.8 $\mathrm{GHz}$ for periodicity $9.9 \mathrm{~mm}$. Investigation of the fields in the full wave simulation revealed that for the smaller periodicity $(6.9 \mathrm{~mm})$ the TM mode has a cutoff at higher frequency than the TE. However, in the case of the array with large periodicity (9.9 $\mathrm{mm}$ ) the cutoff of the TE mode occurs at higher frequencies than the TM. The common TE/TM bandgap in the $\Gamma \mathrm{X}$ direction extends from 15 to $19 \mathrm{GH}$ for the array with periodicity $6.9 \mathrm{~mm}$ and from to 13.8 to $14.9 \mathrm{GHz}$ for the array with periodicity 9.9 $\mathrm{mm}$. The band gap becomes slightly narrower along the XM path of the Brillouin contour.

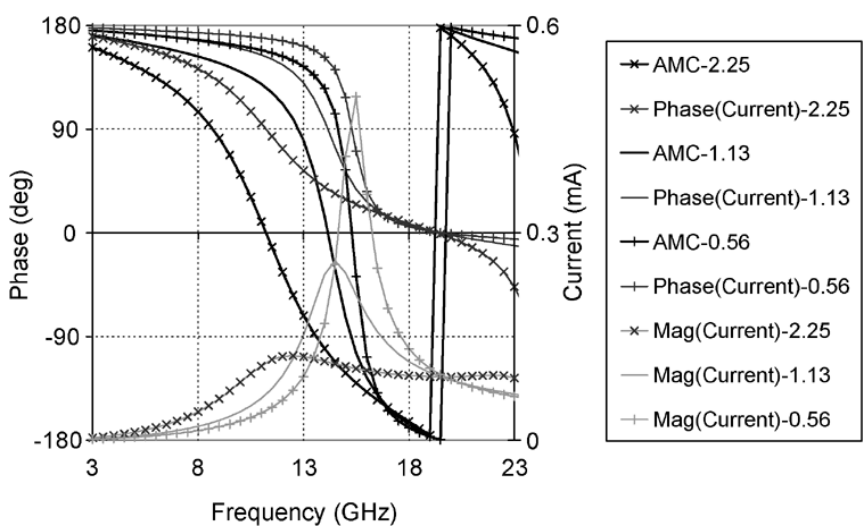

Fig. 9. Current magnitude and phase for the array with periodicity $9.9 \mathrm{~mm}$ and variable substrate thickness.

The analysis of this subsection suggests that the lowest cutoff frequency of the bandgap drops for larger periodicity of the array. This trend follows the reduction of the resonant frequency of the array over the grounded dielectric (i.e., zero current phase) shown in Fig. 4.

\section{Emerging Trends}

Following the investigation on the AMC and EBG characteristics of a metallic periodic array on grounded dielectric substrate, the emerging trend is that as the array periodicity is increased, the AMC frequency goes up, while the EBG frequency drops. These trends are illustrated and quantified in the diagram of Fig. 8, where the AMC and EBG bandwidth for the four 


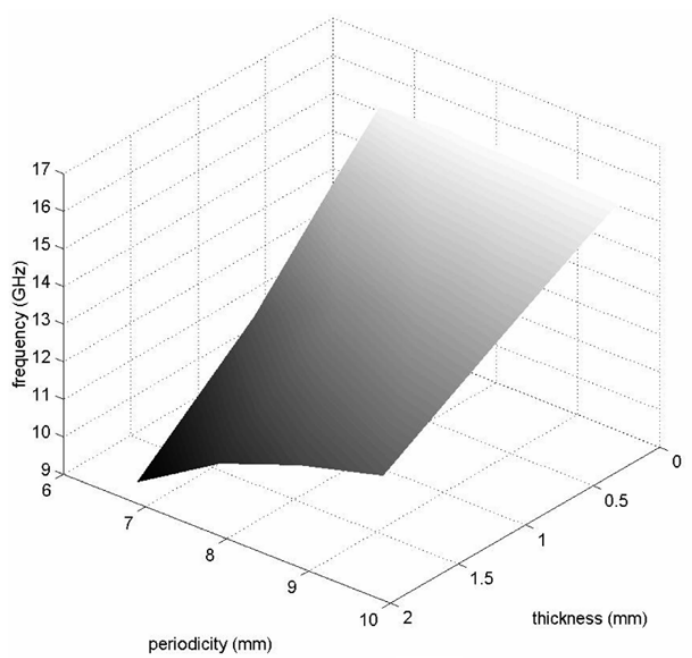

(a)
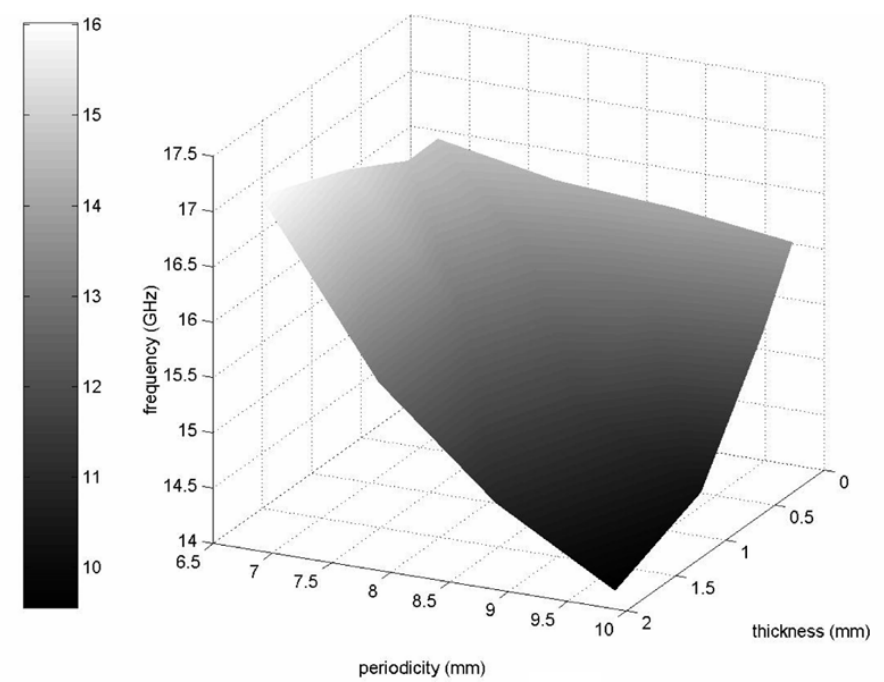

(b)

Fig. 10. (a) Center AMC frequency and (b) lower EBG frequency for square patch arrays printed on grounded dielectric substrate for varying unit cell size $(D)$ and substrate thickness $(S)$. Element size $L=6.1 \mathrm{~mm}$.

arrays under investigation are presented. Here the AMC bandwidth is defined as the frequency range where the fields are reflected with a phase between $-90^{\circ}$ and $90^{\circ}$. Fig. 8 shows the upper and lower frequency for AMC operation and the EBG in the $\Gamma \mathrm{X}$ direction for varying array periodicity, as obtained with full wave MoM method.

As the periodicity of the array increases, the EBG frequency drops. In particular it is evident that the upper cutoff drops with the periodicity linearly. The lower cutoff also drops almost linearly, but for higher periodicities, the TE mode surpasses the TM mode, resulting in narrower common TE/TM bandgap.

Although the array resonance decreases, the AMC operation goes up in frequency for larger unit cells. Assuming a fixed dielectric thickness $S$, the transmission phase value $\phi_{T}$ that satisfies (1) goes up in frequency for larger array periodicity. This explains the AMC trend. As shown in Fig. 8, there is a range of periodicities of the array where the two pairs of curves overlap. This area corresponds to simultaneous AMC and EBG operations in the frequency domain.

\section{EFFECT OF THICKNESS}

A study on the effect of the substrate thickness to the currents induced on the array has been carried out. Fig. 9 shows the current amplitude and phase for the array with periodicity $9.9 \mathrm{~mm}$ lying over a ground plane of variable thickness $(0.5625,1.125$, $2.25,4.5 \mathrm{~mm}$ ). The resonance of the array is almost fixed for all four cases at $19.2 \mathrm{GHz}$. However the frequency of the current peak that corresponds to the AMC operation reduces as the distance to the ground plane is increased, in accordance to the predictions of the resonant cavity model.

The variation of AMC and EBG spectral properties with the geometrical characteristics of the structure (including both thickness and periodicity) is shown in Fig. 10. Fig. 10(a) shows the center AMC for square patch arrays printed on grounded dielectric substrate for varying unit cell size and substrate thickness. Fig. 10(b) shows a similar graph for the lower EBG cutoff. The element size is $L=6.1 \mathrm{~mm}$. The first graph
[Fig. 10(a)] reveals that for thin substrates the effect of the unit cell becomes less important in the AMC response of the structures. For a fixed array unit cell, the AMC frequency reduces almost linearly with the substrate thickness. The linearity is more evident for thicker substrates, while it breaks as the substrate becomes thinner. Furthermore, the slope of reduction is greater for smaller unit cells. Similarly Fig. 10(b) shows that the lower EBG is less sensitive for thin substrates or small periodicities. However the EBG frequency drops for thicker substrates and larger periodicities.

\section{EXPERIMENTAL RESULTS}

To validate our conclusions and demonstrate an AMC surface without vias exhibiting surface wave EBG, the array with unit cell $9.9 \mathrm{~mm}$ has been etched on a dielectric substrate of thickness $1.13 \mathrm{~mm}$ and $\varepsilon_{\mathrm{r}}=2.2$. The overall array consisted of $26 \times 29$ elements. The AMC and EBG properties of the fabricated prototype have been measured. For the EBG measurement, the structure was positioned between absorbing materials. Two horn antennas are used as the transmitter and receiver on either side of the surface. The measured EBG responses are shown in Fig. 11(a). The TM mode has a cutoff at $13.1 \mathrm{GHz}$ and the TE mode at $13.8 \mathrm{GHz}$. A common TE and TM bandgap is found between 13.8 and $14.9 \mathrm{GHz}$ in the $\Gamma \mathrm{X}$ direction and is highlighted in Fig. 11. The absolute band gap is somewhat narrower, 14.4 to $14.9 \mathrm{GHz}$ as shown in the dispersion diagram [Fig. 7(d)]. Very good agreement between measurement and simulation is observed.

The reflection characteristics of the same structure were also measured for normal incidence. The periodicity is small enough for the grating lobe region to be well above $20 \mathrm{GHz}$. The reflection phase of the AMC is taken in the far field using horn antennas and is then normalized with respect to an identical measurement in the absence of the array. The simulated and measured AMC responses of this structure are shown in Fig. 11(b). Note that there are 2 pairs of curves for the AMC measurement and simulation. This is due to the finite thickness 


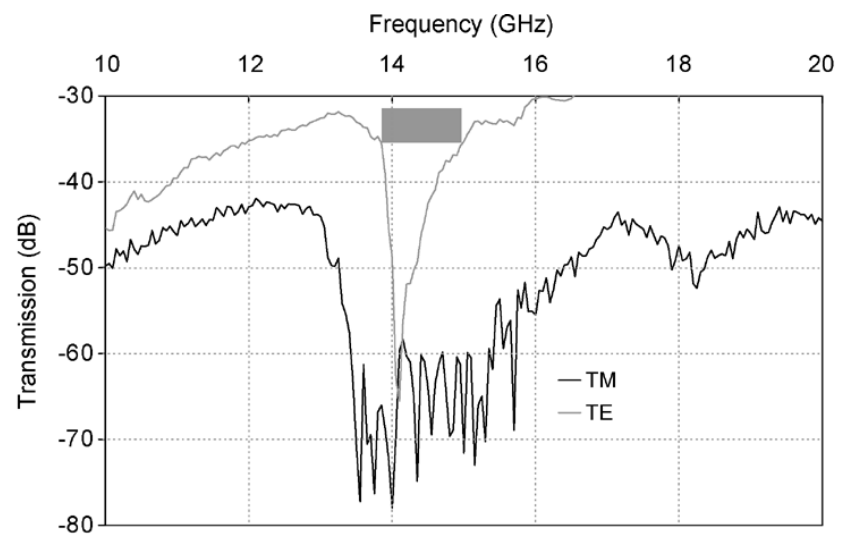

(a)

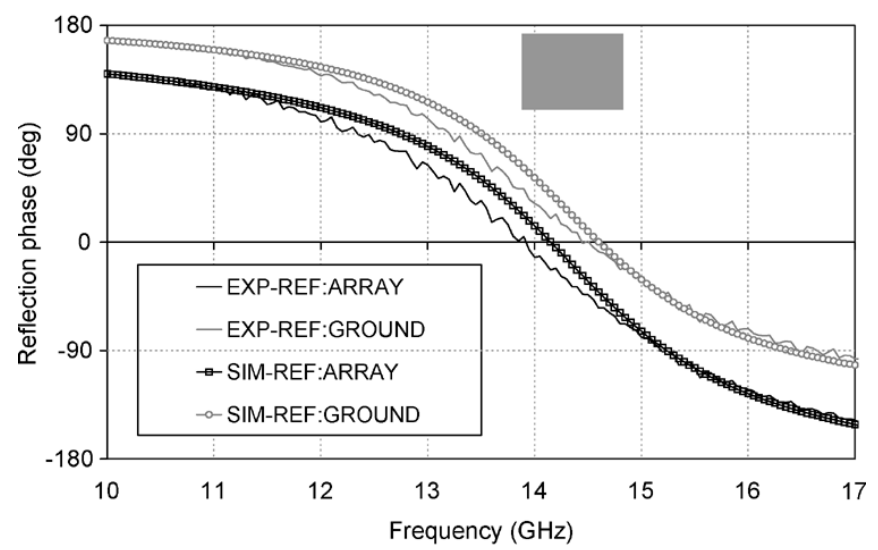

(b)

Fig. 11. (a) Measured EBG performance for square patch array. In mm: patch 6.1, unit cell 9.9, dielectric thickness 1.15 ( $\mathrm{er}=2.2$ ). Shaded is the bandgap region $(\Gamma \mathrm{X})$. (b) Measured AMC response for square patch array. In mm: patch 6.1 , unit cell 9.9, dielectric thickness $1.15(\mathrm{er}=2.2)$. Shaded is the bandgap region $(\Gamma \mathrm{X})$.

of the AMC structure and hence the two different possible definitions of the reflection reference plane. This can be either the plane of the array or the ground. Thus, one result is taken assuming reflection at the array plane (REF:ARRAY) and another assuming reflection at the ground plane (REF:GROUND). In both cases, very good agreement between simulated and experimental results is observed. The AMC band $\left(-90^{\circ}\right.$ to $\left.90^{\circ}\right)$ lies between $13.3-16.45 \mathrm{GHz}$ and $12.55-15.2 \mathrm{GHz}$ respectively. The measured band gap region is highlighted in the graph. Simultaneous AMC and EBG (ГX) operation of $1.1 \mathrm{GHz}$ ( $\sim 7.5 \%$ ) bandwidth has been achieved.

\section{CONCLUSION}

The AMC and EBG characteristics of periodic metallic arrays printed on grounded dielectric substrate in the absence of vias have been studied. The currents excited on the array elements for normal and oblique incidence have been investigated. It has been demonstrated that the AMC operation is not directly related to the array resonance, but rather to a cavity resonance. This explains why the AMC frequency increases with periodicity while the FSS resonance reduces. On the contrary the EBG occurs by virtue of the array resonance and hence the EBG spectral properties follow those of the FSS array. The opposite spectral dependence of the AMC and EBG properties on the array periodicity provides the flexibility to tailor the array characteristics according to the application. The effect of substrate thickness has also been assessed. In order to demonstrate our findings, a uniplanar array with simultaneous AMC and EBG responses in a $7.5 \%$ fractional bandwidth has been fabricated and measured. The uniplanar arrays offer the advantage of simple fabrication and easier scalability to higher frequencies.

\section{REFERENCES}

[1] P. De Maagt, R. Gonzalo, J. C. Vardaxoglou, and J. M. Baracco, "Photonic bandgap antennas and components for microwave and (sub)millimeter wave applications," IEEE Trans. Antennas Propag., Special Issue on Metamaterials, vol. 51, no. 10, pp. 2667-2677, Oct. 2003.

[2] D. Sievenpiper, Z. Lijun, R. F. Broas, N. G. Alexopoulos, and E. Yablonovitch, "High-impedance electromagnetic surfaces with a forbidden frequency band," IEEE Trans Microw. Theory Tech., vol. 47, no. 11, pp. 2059-2074, Nov. 1999.

[3] A. P. Feresidis, G. Apostolopoulos, N. Serfas, and J. C. Vardaxoglou, "Closely Coupled Metallodielectric Electromagnetic Band Gap (CCMEBG) structures formed by double layer dipole and tripole arrays," IEEE Trans. Antennas Propag., vol. 52, no. 5, pp. 1149-1158, May 2004.

[4] R. Abhari and G. V. Eleftheriades, "Metallo-dielectric electromagnetic bandgap structures for suppression and isolation of the parallel-plate noise in high-speed circuits," IEEE Trans. Microw. Theory Tech., vol. 51, no. 6, pp. 1629-1639, Jun. 2003.

[5] R. Gonzalo, P. de Maagt, and M. Sorolla, "Enhanced patch antenna performance by suppressing surface waves using photonic bandgap substrates," IEEE Trans. Microw. Theory Tech., vol. 47, no. 11, pp. 2131-2138, Nov. 1999.

[6] F. Yang and Y. Rahmat-Samii, "Microstrip antennas integrated with electromagnetic band-gap (EBG) structures: a low mutual coupling design for array applications," IEEE Trans. Antennas Propag., vol. 51, no. 10, pp. 2936-2946, Oct. 2003.

[7] Y. R. Lee, A. Charaya, D. S. Lockyer, and J. C. Vardaxoglou, "Dipole and tripole metallodielectric photonic bandgap (MPBG) structures for microwave filter and antenna applications," Proc. Inst. Elect. Eng. Optoelectron., vol. 127, no. 6, pp. 395-400, Dec. 2000.

[8] F.-R. Yang, K.-P. Ma, Y. Qian, and T. Itoh, "A novel TEM waveguide Using Uniplanar Compact Photonic-Bandgap (UC-PBG) structure," IEEE Trans. Microw. Theory Tech., vol. 47, no. 11, pp. 2092-2098, Nov. 1999.

[9] H. Mosallaei and K. Sarabandi, "Antenna miniaturization and bandwidth enhancement using a reactive impedance substrate," IEEE Trans. Antennas Propag., vol. 52, no. 9, pp. 2403-2414, Sep. 2004.

[10] Y. E. Erdemli, K. Sertel, R. A. Gilbert, D. E. Wright, and J. L. Volakis, "Frequency-selective surfaces to enhance performance of broad-band reconfigurable arrays," IEEE Trans. Antennas Propag., vol. 50, no. 12, pp. 1716-1724, Dec. 2002.

[11] S. Wang, A. Feresidis, G. Goussetis, and J. C. Vardaxoglou, "Low profile resonant cavity antenna with artificial magnetic conductor ground plane," IEE Electron. Lett., vol. 40, no. 7, pp. 405-406, April 2004.

[12] A. P. Feresidis, G. Goussetis, S. Wang, and J. C. Vardaxoglou, "Artificial magnetic conductor surfaces and their application to low profile highgain planar antennas," IEEE Trans. Antennas Propag., Special Issue on AMC, Soft Hard Surfaces and Other Complex Surfaces, vol. 53, no. 1, pp. 209-215, Jan. 2005.

[13] Y. Zhang, J. von Hagen, M. Younis, C. Fischer, and W. Wiesbeck, "Planar Artificial Magnetic Conductors and Patch Antennas," IEEE Trans. Antennas Propag., Special Issue on Metamaterials, vol. 51, no. 10, pp. 2704-2712, Oct. 2003.

[14] R. Mittra, C. H. Chan, and T. Cwik, "Techniques for analyzing frequency selective surfaces-a review," Proc. IEEE, vol. 76, no. 12, pp. 1593-1615, Dec. 1988.

[15] J. C. Vardaxoglou, Frequency Selective Surfaces Analysis and Design. New York: Wiley, 1997.

[16] S. Maci, M. Caiazzo, A. Cucini, and M. Casaletti, "A network theory for EBG surfaces composed by FSS printed on a grounded dielectric slab," IEEE Trans. Antennas Propag., Special Issue on AMC, Soft Hard Surfaces and Other Complex Surfaces, Jan. 2005.

[17] A. P. Feresidis and J. C. Vardaxoglou, "High gain planar antenna using optimized partially reflective surfaces," Proc. Inst. Elect. Eng. Microw. Antennas Propag., vol. 148, no. 6, pp. 345-350, Dec. 2001. 
[18] A. C. Lima and E. A. Parker, "Fabry-Perot approach to the design of double layer FSS," Proc. Inst. Elect. Eng. Microw. Antennas Propag., vol. 143, no. 2, pp. 157-162.

[19] Y. Guo, G. Goussetis, A. P. Feresidis, and J. C. Vardaxoglou, "Efficient modeling of novel uniplanar left-handed metamaterials," IEEE Trans. Microw. Theory Tech, vol. 53, no. 4, pp. 1462-1468, Apr. 2005.

[20] R. E. Collin, Field Theory of Guided Waves. New York: IEEE Press, 1991.

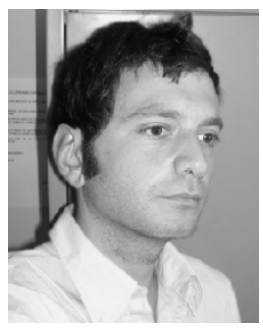

George Goussetis (M'01) was born in Athens, Greece, in 1976. He received the Electrical and Computer Engineering degree from the National Technical University of Athens, in 1998, the B.Sc. degree in physics from University College London (UCL), London, U.K., in 2002, and the Ph.D. degree in the area of waveguide filters from the University of Westminster, London, in 2002.

In 1998, he joined the Space Engineering Spa, Roma, Italy as a trainee RF Engineer. In 1999, he joined the Wireless Communications Research Group, University of Westminster, as a Research Assistant. In 2002, he joined the Wireless Communications Research Group, Loughborough University, U.K., where he is currently employed as a Senior Research Fellow. He has published more than 50 peer reviewed journals and conference papers. His research interests include the modeling and design of microwave filters, properties of periodic structures as well as frequency domain numerical techniques.

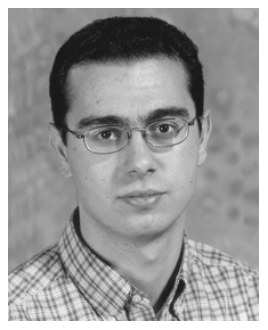

Alexandros P. Feresidis (M'98) was born in Thessaloniki, Greece, in 1975. He received the B.Sc. degree in physics from Aristotle University of Thessaloniki (AUTH), in 1997, the M.Sc.(Eng.) degree in radio communications and high-frequency engineering from the University of Leeds, Leeds, U.K., in 1998, and the Ph.D. degree in electronic and electrical engineering from Loughborough University, Leicestershire, U.K., in 2002.

During the first half of 2002, he was a Research Associate with the Wireless Communications Research Group, Department of Electronic and Electrical Engineering, Loughborough University. Since July 2002, he has been a Lecturer in the same department. His research interests include analysis and design of electromagnetic band gap (EBG) materials and frequency selective surfaces (FSS), metamaterial structures, array antennas, base station antennas, computational electromagnetics and microwave circuits. He has published over 50 refereed journal and conference proceeding papers.

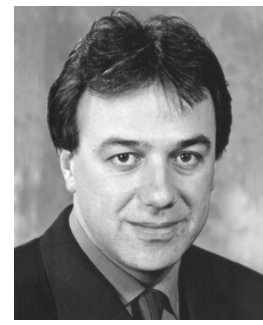

John (Yiannis) C. Vardaxoglou (M'87) received the B.Sc. degree in mathematics (mathematical physics) and the Ph.D. degree from the University of Kent at Canterbury, U.K., in 1981 and 1985, respectively.

In January 1988, he was appointed Lecturer in Communications with the Department of Electronic and Electrical Engineering, Loughborough University of Technology, U.K. He was promoted to the position of Senior Lecturer in January 1992. In 1998, he was appointed Professor of Wireless Communications. He holds the Chair of Wireless Communications at Loughborough University and is the Founder of the Centre for Mobile Communications Research (CMCR). He established the Antennas and Microwaves Research Group at Loughborough University and he heads the Centre for Mobile Communications Research (CMCR). He has been active in the area of electromagnetic modeling and applications of frequency selective surfaces (FSS). His current research interests include wireless communication networks, array antennas, FSS, radomes, leaky-wave resonant antennas, optical control of microwaves and devices, periodic surfaces and EBG/PBG materials, and mobile telephone antennas. His contribution to the CMCR lies in the analysis and design of small-loaded antennas for mobile telephony. He has served as a Consultant to various industries in the U.K. and abroad. He holds three patents and is the Technical Director of Antrum Ltd. He has published over 130 refereed journals and conference proceeding papers and has written a research monograph on frequency selective surfaces.

Dr. Vardaxoglou is currently the Chairman of the executive committee of the IEE's Antennas and Propagation Professional Network in and he chairs the IEEE's distinguish lecturer program of the Antennas and Propagation society. He Chairs the Executive Committee of Metamorphose, EU FP6 Network of Excellence of Metamaterials. He chaired the 1st and 2nd IEE Antenna Measurements and SAR (AMS'02 and AMS'04) conferences and has been in the organizing committee of the 2001 and 2003 IEE International Conferences on Antennas and Propagation. He was the general Chair of the 1st Loughborough Antennas and Propagation Conference (LAPC'05) 